\title{
Capacidad de Agencia de Autocuidado en Personas con Hipertensión Arterial hospitalizadas en una Clínica de Bogotá, Colombia
}

Luz N. Rivera Álvarez

Enfermera. M. Sc. en Enfermería. Especialista en Enfermería en Unidad de Cuidado Intensivo. Facultad de Enfermería, Universidad Nacional de Colombia. Unidad de cuidado Intensivo, Clínica Country. Bogotá, Colombia. E-mail: Inriveraa@unal.edu.co

Recibido 28 Marzo 2006/Enviado para Modificación 24 Agosto 2006/Aceptado 4 Octubre 2006

\section{RESUMEN}

Objetivo Describir la capacidad que tiene la persona hospitalizada con diagnóstico de hipertensión arterial para realizar actividades de cuidado hacia sí mismo.

Metodología Se utilizó un diseño descriptivo, con abordaje cuantitativo que permitió evaluar la capacidad de agencia de autocuidado en 90 personas hospitalizadas con diagnostico de hipertensión arterial en una clínica de Bogotá, mediante el empleo de la escala ASA "Apreciación de la agencia de autocuidado" de Evers e Isenberg.

Resultados Los resultados muestran que el $53 \%$ de las personas hospitalizadas con hipertensión arterial tuvieron deficiente capacidad de agencia para el autocuidado en tanto que el $47 \%$ presentaron suficiente capacidad de agencia para el autocuidado. En cuanto a los ítems del instrumento apreciación de la capacidad de agencia de autocuidado (ASA) que obtuvieron los más altos puntajes, se encontró que los participantes presentaron capacidades para: Máxima atención y vigilancia, motivación o metas orientadas hacia el autocuidado, razonamiento dentro de un marco de referencia de autocuidado, habilidades (cognitivas, de percepción, de comunicación e interpersonales) para el autocuidado e integración de las operaciones de autocuidado con otros aspectos de la vida.

Conclusiones El cuidado de personas hipertensas es un reto para el personal de salud y la comunidad; los cuidados de enfermería deben estar enfocados a ayudar al individuo a llevar a cabo y mantener por si mismo, acciones de autocuidado para conservar la salud y la vida, recuperarse de la enfermedad y afrontar las consecuencias de esta.

Palabras Clave: Autocuidado, enfermería, teoría de enfermería (fuente: DeCS, BIREME). 


\section{ABSTRACT \\ Self-care agency ability in people suffering from high blood pressure hospitalised in Bogotá, Colombia}

Objective Describing how hospitalised people who have been diagnosed as having high blood pressure carry out self-care activities.

Methodology A descriptive design, having a quantitative approach, was used for evaluating the self-care agency ability of 90 people who had been hospitalised in Bogotá diagnosed as suffering from high blood pressure. Evers and Isenberg's SAA (self-care agency appreciation) scale was used. Results The results showed that $53 \%$ of people hospitalised suffering from high blood pressure had deficient SAA. Regarding the SAA instrument's items obtaining the highest scores, it was found that the participants were able to maximise attention and vigilance, had motivation or self-careorientated goals, reasoned within a self-care reference framework, had self-care abilities (cognitive, perception, communication and interpersonal) and integrated self-care operations with other aspects of life.

Conclusions Caring for hypertense people is a challenge for health-care personnel and the whole community. Nursing care should focus on helping individuals to look after themselves, engage in self-care action for conserving their health and lives, recover from illness and confront its consequences.

Key Words: Self-care, nursing, nursing theory (source: $M e S H, N L M$ ).

$\mathrm{E}$ l ser humano como fuente integradora de aspectos emocionales, intelectuales y sociales, desarrolla estrategias necesarias para la conservación de su estructura y funcionalidad, contribuyendo cada vez a un mejor nivel y calidad de vida. Una de estas estrategias es el autocuidado que puede verse afectado cuando el individuo se encuentra ante el riesgo o la presencia de sufrir hipertensión arterial. La situación de enfermedad puede generar modificaciones en los estilos de vida y factores psicosociales como la depresión, hostilidad, ansiedad, aislamiento social y estrés (1), que pueden influir en la ocurrencia de un evento coronario o la muerte, por ende desarrolla el individuo un autocuidado adquirido para conservar la salud, cuidarla y protegerla.

El tratamiento de la hipertensión arterial esta caracterizado por el marcado impacto emocional, los cambios necesarios en el estilo de vida y la capacidad de agencia de autocuidado que el individuo y su familia requieren para minimizar los factores de riesgo cardiovascular; dentro de las medidas protectoras como parte del tratamiento de la hipertensión arterial, se encuentran: la realización de 
ejercicio físico, adaptación a nuevos regímenes nutricionales, peso ideal, disminución del consumo de licor y del cigarrillo, adherencia al tratamiento farmacológico y el manejo de respuestas psicológicas.

El autocuidado como valor inherente a cada ser humano y percibido esté como un deber y un derecho para consigo mismo y con la sociedad, es una estrategia que responde a las metas y prioridades de enfermería, que toma la tendencia hacia el autoconocimiento y empoderamiento del individuo y de la sociedad de su propia salud y a la vez reconoce la presencia de factores culturales, de índole educativo y socioeconómico que condicionan la agencia de autocuidado e influye en el autocuidado.

Además, el autocuidado puede considerarse como un complemento de los servicios de salud ofrecidos por los profesionales, donde se comparte la responsabilidad con el usuario, como herramienta útil para la promoción de salud y prevención de eventos coronarios como el Infarto agudo de Miocardio y la Angina de pecho y /o evento cerebro vascular, situaciones de alteración de la salud que generan deterioro de la calidad de vida, que conllevan a un alto costo tanto económico como social.

Por otro lado, el autocuidado por ser un tema que concierne al ser humano en general, ha retomado fuerza en la sociedad actual, que insta a la enfermera a fortalecer y crear aportes investigativos frente al tema, a buscar la validación de conceptos y de hallazgos investigativos dentro de la Teoría del déficit de autocuidado de la enfermera Dorothea Orem.

Capacidad de agencia de autocuidado

Orem introdujo el concepto de agencia de autocuidado que refleja las capacidades individuales para atender los requisitos del autocuidado.

La agencia de autocuidado es definida por Orem (2) como la «compleja capacidad desarrollada que permite, a los adultos y adolescentes en proceso de maduración, discernir los factores que deben ser controlados o tratados para regular su propio funcionamiento y desarrollo, para decidir lo que puede y debería hacerse con respecto a la regulación, para exponer los componentes de su demanda de autocuidado terapéutico (requisitos de autocuidado, tecnologías, medidas de cuidados) y finalmente, para realizar las actividades de cuidado determinadas para cubrir sus requisitos de autocuidado a lo largo del tiempo"(3).

La agencia de autocuidado puede estudiarse en relación con las capacidades del individuo como ser integral, racional, biológico y emocional, que entran en 
juego cuando el ser humano realiza algún tipo de acción incluyendo su repertorio de habilidades y la clase de conocimiento sobre su salud para ocuparse de una gama de conductas concretas.

Los tipos de habilidades para tener una agencia de autocuidado que han sido desarrolladas por Orem y el Grupo de la Conferencia de Desarrollo de Enfermería - NDCG (Nursing Development Conference Group) son (4):

- Las capacidades fundamentales y disposición de autocuidado. Consisten en habilidades básicas del individuo como la sensación, percepción, memoria y orientación.

- Los componentes de poder (capacidades que facilitan el autocuidado). Son capacidades específicas que se relacionan con la habilidad del individuo para comprometerse en el autocuidado, la valoración de la salud, energía y conocimiento del autocuidado.

- Capacidades para operaciones de autocuidado. Son las habilidades del ser humano más inmediatas;

Algunas de las características para ser participante creativo en el autocuidado es la comprensión de los acontecimientos del entorno, una actitud reflexiva sobre los pensamientos saludables que se dan por sí mismo en el humano, escuchar, escribir las reflexiones que escucha o que se hace de sí mismo y realizar una continua autoevaluación sobre los hábitos de autocuidado y las necesidades de cambio.

\section{METODOLOGÍA}

Durante cinco meses se estudiaron 90 pacientes hospitalizados en los diferentes servicios de hospitalización en una clínica de Bogotá. El diseño de la investigación fue de tipo descriptivo, con abordaje cuantitativo que permitió describir la capacidad que tiene la persona hospitalizada con diagnóstico de hipertensión arterial para realizar actividades de cuidado hacia sí mismo, es decir, la capacidad de agencia de autocuidado.

Los pacientes, seleccionados por muestreo intencional, debían cumplir los siguientes criterios: Mujeres y hombres adultos mayores de 18 años con diagnóstico de hipertensión arterial confirmado, hospitalizados en los diferentes servicios de hospitalización de una clínica de Bogotá, consciente, que pueda comunicarse y voluntariamente acepte contestar la encuesta y que su estado de salud le permita el diligenciamiento. 
Para la investigación, se empleó la escala ASA “Apreciación de la agencia de autocuidado", está escala fue el producto del primer trabajo colaborativo, que comenzó en 1983 cuando Isenberg fue invitada como consultora a la Facultad de Ciencia de la Salud de la Universidad Mastricht, en Holanda, para desarrollar investigaciones basadas en teoría de enfermería. El equipo que participo en el desarrollo y prueba psicométrica de esta escala incluyó a los profesores Hans Philipsen, George Evers, Ger Brauns, Harriet Smeets e Isenberg (5)

La escala ASA ha sido traducida en varios idiomas, en países de Europa, Asia oriental y en Latinoamérica y validado el instrumento en cada cultura. Este proyecto colaborativo le dio al equipo la oportunidad de identificar conocimiento universal de enfermería y por medio de comparación transcultural, identificar conocimientos culturalmente específico. La escala consta de 24 ítems, en una escala Likert de 4 puntos (siempre, casi siempre, casi nunca y nunca), para un rango real de 24 a 96 puntos por escala.

La validez de constructo de la escala ASA, fue estudiada por Evers e Isenberg, la agencia de autocuidado se relaciono positivamente con el estado de salud (6). La validez externa se demostró con grupos muestrales de diferentes países con características similares en cuantos a sus capacidades de autocuidado quienes obtuvieron medias semejantes al responder la escala. La confiabilidad del instrumento fue mayor de 0.70 en casi la totalidad de los estudios (7).

Esther Gallegos confirmó los componentes de poder como habilidades específicas que se mueven para ejecutar operaciones de autocuidado y la relación teórica entre los factores condicionantes básicos y la capacidad de agencia de autocuidado, con una confiabilidad de 0,77 de la escala “apreciación de la agencia de autocuidado” ASA, aplicada está en una población mexicana en versión español; de igual manera, los resultados de está investigación confirmaron la validez de constructo del instrumento.

\section{Procedimiento}

La recolección de datos se realizó entre los meses de Diciembre del 2004 a Abril del 2005, en los servicios de hospitalización de una clínica en Bogotá.

Los participantes en la investigación fueron personas adultas hospitalizadas con diagnóstico de hipertensión arterial confirmada. Se explicó a cada persona el objetivo de la investigación junto con el consentimiento de participación en el estudio y se garantizó la absoluta reserva de la información suministrada. 
Posteriormente, el participante procedía a diligenciar la escala "apreciación de la agencia de autocuidado", sin participación directa del investigador, quien recogía la escala 30 minutos después y resolvía inquietudes sobre el diligenciamiento.

Finalizado el proceso de recolección de datos, se introdujo la información recopilada de la Escala "Apreciación de la capacidad de agencia de autocuidado”ASA, en una hoja de cálculo utilizando el programa de Excel. Se determinó el puntaje total de cada ítem, calculando el valor de la respuesta (que va de 1 a 4 en la escala Likert) por el número de participantes, para una puntuación total de 360 puntos. De esta forma, se analizó cada ítem de la Escala ASA, con los planteamientos de la teórica Dorothea Orem.

\section{RESULTADOS}

Descripción demográfica

El $60 \%$ de los participantes pertenecen al género femenino con una edad media de 60 años, con una edad mínima de 31 años y una edad máxima de 77 años y el $40 \%$ de los participantes correspondieron al genero masculino con una edad media de 56 años, en los rangos de 32 a 76 años.

Con respecto a la edad, las personas con hipertensión arterial se ubicaron en el rango de edad mayor de 60 años (49\%), dato congruente con el comportamiento de la hipertensión arterial a medida que avanza la edad, el 39 $\%$ de las personas hipertensas se encontró en el rango de edad de 46 a 60 años y un $12 \%$ de los individuos pertenecían al rango de edad de los 30 a 45 años, personas que aun se encuentran en su etapa productiva.

Capacidad de agencia de autocuidado en personas con hipertensión arterial

La media resultante de la capacidad de agencia de autocuidado en los participantes correspondió a 716, con una desviación estándar de 73 , lo que significa que los resultados estuvieron algo dispersos, además, la encuesta con puntuación más baja fue de 55 puntos y la más alta fue de 90 puntos.

Según los resultados globales arrojados de la Escala ASA se encontró que:

- $\quad$ El 53 \% de las personas hospitalizadas con HTA presentaron Deficiente capacidad de agencia para el autocuidado.

- $\quad$ El 47 \% de las personas hospitalizadas con HTA tuvieron Suficiente capacidad de agencia para el autocuidado. 
Descripción por ítem de la escala ASA en personas hospitalizadas con HTA

Ahora bien, con el objeto de conocer los aspectos que pueden orientar las intervenciones de enfermería que busquen potencializar la agencia de autocuidado en las personas con hipertensión arterial, en la tabla 1 se describen a continuación los ítems de la escala «Apreciación de la agencia de autocuidado», con mejor puntaje:

Tabla 1. Porcentaje de los Capacidades operativas de la "Agencia de autocuidado" en personas con hipertensión arterial

\begin{tabular}{lcccc}
\multicolumn{1}{c}{ Item } & Siempre & $\begin{array}{c}\text { Casi } \\
\text { Siempre }\end{array}$ & $\begin{array}{c}\text { Casi } \\
\text { Nunca }\end{array}$ & Nunca \\
\hline $\begin{array}{l}\text { Puedo hacer lo necesario para mantener limpio mi } \\
\text { alrededor }\end{array}$ & 84 & 14 & 2 & 0 \\
$\begin{array}{l}\text { Puedo conseguir la información necesaria si mi } \\
\text { salud se ve amenazada }\end{array}$ & 78 & 11 & 11 & 0 \\
$\begin{array}{l}\text { Con tal de mantenerme limpio, puedo cambiar la } \\
\text { frecuencia con que me baño }\end{array}$ & 79 & 14 & 4 & 3 \\
$\begin{array}{l}\text { Soy capaz de tomar medidas para asegurar que mi } \\
\text { familia yo estemos seguros }\end{array}$ & 68 & 24 & 8 & 0 \\
$\begin{array}{l}\text { Puedo buscar ayuda si no puedo cuidarme yo } \\
\text { misma (o) }\end{array}$ & 60 & 32 & 8 & 0 \\
$\begin{array}{l}\text { Si tengo que tomar un nuevo medicamento, puedo } \\
\text { obtener información sobre los efectos indeseables }\end{array}$ & 57 & 13 & 8 & 22 \\
$\begin{array}{l}\text { Soy capaz de valorar que tan efectivo es lo que } \\
\text { hago para mantenerme con salud }\end{array}$ & 55 & 25 & 16 & 3 \\
$\begin{array}{l}\text { Puedo hacer cambios o ajustes si tengo problemas } \\
\text { para moverme }\end{array}$ & 54 & 27 & 13 & 6 \\
$\begin{array}{l}\text { Tengo amigos de mucho tiempo a quienes puedo } \\
\text { recurrir cuando necesito ayuda }\end{array}$ & 53 & 24 & 19 & 4 \\
\hline
\end{tabular}

De acuerdo con la teoría de Orem, los componentes de poder son las capacidades específicas que se relacionan con la habilidad del individuo para comprometerse en el autocuidado, los ítems del instrumento ASA, mejor calificados por los pacientes hipertensos hospitalizados están relacionadas con los componentes de poder: máxima atención y vigilancia, motivación o metas orientadas hacia el autocuidado, razonamiento dentro de un marco de referencia de autocuidado, repertorio de habilidades (cognitivas, de percepción, de comunicación e interpersonales) para el autocuidado e integración de las operaciones de autocuidado con otros aspectos de la vida. Los resultados reflejan que las personas con hipertensión arterial del estudio satisfacen los siguientes requisitos de autocuidado en estado de alteración de la salud:

- Buscar y asegurar asistencia medica apropiada

- Tener conciencia de y atender a los efectos de las condiciones patológicas, en este caso la hipertensión arterial. 
- Modificar el autoconcepto y la autoimagen como aceptación del propio Estado de salud y necesidades de cuidado de la salud.

- Aprender a vivir con los efectos de las condiciones patológicas y las medidas terapéuticas necesarias, en un camino que promueva el desarrollo personal continuo.

Con el objeto de elaborar planes de cuidado de enfermería dirigido a personas hipertensas, en el sistema de enfermería de apoyo educativo (planteado por Orem), es necesario identificar las necesidades y las dificultades que presentan las personas que viven con HTA para lograr ser un buen agente de autocuidado (Tabla 2).

Tabla 2. Porcentaje de los ítems de la escala ASA con menor puntuación en personas hospitalizadas con HTA

\begin{tabular}{|c|c|c|c|c|}
\hline Item & Siempre & $\begin{array}{l}\text { Casi } \\
\text { Siempre }\end{array}$ & $\begin{array}{l}\text { Casi } \\
\text { Nunca }\end{array}$ & Nunca \\
\hline Puedo tomar tiempo para mí. & 10 & 23 & 33 & 34 \\
\hline $\begin{array}{l}\text { Puedo dormir lo suficiente para sentirme } \\
\text { descansado. }\end{array}$ & 13 & 22 & 38 & 27 \\
\hline $\begin{array}{l}\text { Con la actividad diaria puedo tomar tiempo para } \\
\text { cuidarme. }\end{array}$ & 17 & 20 & 33 & 30 \\
\hline $\begin{array}{l}\text { Verifico si las formas habituales de mantenerme } \\
\text { con salud son buenas. }\end{array}$ & 14 & 29 & 35 & 22 \\
\hline $\begin{array}{l}\text { Pienso en revisar mi cuerpo para saber si hay } \\
\text { algún cambio. }\end{array}$ & 14 & 27 & 17 & 42 \\
\hline $\begin{array}{l}\text { A veces pienso en hacer ejercicio y descansar } \\
\text { un poco durante el día, y lo hago. }\end{array}$ & 24 & 18 & 36 & 22 \\
\hline $\begin{array}{l}\text { Con frecuencia no tengo la energía para } \\
\text { cuidarme como debo. }\end{array}$ & 16 & 39 & 27 & 18 \\
\hline $\begin{array}{l}\text { Puedo alimentarme de manera que mi peso se } \\
\text { mantenga }\end{array}$ & 34 & 23 & 20 & 23 \\
\hline
\end{tabular}

Los ítems del instrumento apreciación de la capacidad de agencia de autocuidado, menos calificados por los pacientes HTA hospitalizados están relacionados con los componentes de poder citados por Orem: uso controlado de la energía física, para la iniciación y continuación de las operaciones de autocuidado; control de la posición del cuerpo y sus partes, toma de decisiones sobre autocuidado y la operacionalización de estas decisiones; adquisición, conservación y operacionalización técnica sobre autocuidado y orden discreto de las acciones de autocuidado.

Los resultados reflejan que las personas con HTA del estudio no satisfacen los siguientes requisitos de autocuidado en estado de alteración de la salud: 
- Llevar a cabo efectivamente las prescripciones médicas diagnosticas, el tratamiento y las medidas de rehabilitación, dirigido a prevenir otro tipo de patologías (enfermedades coronarias o eventos cerebrovasculares) o a la patología misma (hipertensión arterial).

- Ser consciente de dar atención a la regulación de los efectos secundarios del tratamiento médico.

Además, se encontró que en las personas hipertensas del estudio existe una insatisfacción del requisito universal de autocuidado, que hace referencia al mantenimiento del equilibrio entre la actividad y el reposo.

\section{DISCUSIÓN}

La agencia de autocuidado ha sido definida como la capacidad que tiene el individuo para tomar decisiones sobre su salud y la responsabilidad de su propio cuidado; y en la medida que se mejoran estas capacidades de agencia de autocuidado en las personas que viven con hipertensión arterial se promueve la satisfacción de los requisitos de autocuidado en estados de desviación de la salud y a su vez la promoción de la salud y bienestar de está población y la prevención de enfermedades coronarias y eventos cerebrovasculares. Esto implica para el profesional de enfermería, la creación de programas que promuevan el autocuidado en las personas con hipertensión arterial basados en la teoría del déficit de autocuidado de Orem.

La mayoría de los participantes consideran que siempre pueden hacer lo necesario para mantener limpio su alrededor, Orem en sus escritos sobre el meta paradigma ambiente hace referencia a los factores físicos, químicos y biológicos, estos últimos incluyen el control de organismos o agentes infecciosos. Este ítem hace referencia al primer componente de poder que enuncia la capacidad que tiene el individuo para prestar atención y vigilancia a las condiciones internas y externas y a factores significativos para el autocuidado (8).

Los participantes del estudio afirman que pueden conseguir la información necesaria, si su salud se ve amenazada; de acuerdo con Orem el conocimiento es una de las capacidades fundamentales y de disposición para el autocuidado, que permite el logro de los siguientes requisitos de autocuidado en estado de alteración de la salud: 1 . Tener conciencia de y atender a los efectos de las condiciones patológicas y, 2. Llevar a cabo efectivamente las prescripciones 
médicas diagnosticas, el tratamiento y las medidas de rehabilitación específicas para la enfermedad. Para Orem, la enfermera esta caracterizada por sus conocimientos y su capacidad para usar sus conocimientos y habilidades para producir enfermería en una variedad de situaciones y ser agente transmisor de dichos conocimientos.

Para Debra (9) la información es importante para que el paciente con enfermedad cardiovascular logre el control de sus sentimientos y actitudes como también disminución de factores psicológicos como la ansiedad, el estrés y la hostilidad, además el mantener informado al individuo y a la familia acerca de su patología, medidas terapéuticas, como el control de factores de riesgo permite la participación activa de su autocuidado.

El cuidado de los profesionales, debe estar orientado en el sistema de enfermería de apoyo educativo que menciona Orem y hacer hincapié en suministrar información al individuo y a la familia, para que puedan apropiarse oportunamente y con soporte efectivo, del cuidado de la persona hipertensa durante la hospitalización y posteriormente en casa.

En general, las personas hipertensas pueden buscar ayuda si no pueden cuidarse por sí mismas y toman las medidas necesarias para asegurar que la familia y el individuo estén seguros. Orem elaboro un triangulo jerárquico para explicar la estructura substantiva de la agencia de autocuidado, allí menciona las "capacidades para operaciones de autocuidado" como las habilidades del ser humano más inmediatas, es decir, primero el individuo investiga condiciones y factores de sí mismo y del medio ambiente que son significativos para su autocuidado (operaciones estimativas); posteriormente el individuo toma juicios y decisiones (operaciones Transicionales), en el caso de que el individuo no puede cuidarse por sí mismo, él toma la decisión de buscar ayuda en su núcleo familiar, sus redes sociales y en los profesionales de salud, que le ayuden a cubrir o a encontrar la demanda terapéutica de autocuidado y el desarrollo de su agencia de autocuidado; finalmente el individuo construye medidas para satisfacer los requisitos de autocuidado por sí mismo (operaciones productivas)

En la pregunta "tengo amigos de mucho tiempo a quienes puedo recurrir cuando necesito ayuda”; es evidente el soporte social para la persona con hipertensión arterial como agente de autocuidado, el cual esta relacionada con el respaldo, motivación y apoyo para comprometerse con su propio cuidado, que contribuye a minimizar el estrés y la ansiedad. Vargas Rosero en su trabajo 
de grado de Maestría en enfermería, estudio fenomenológico, concluye que las personas con enfermedad cardiovascular que logran modificar conductas de riesgo cuentan con personas significativas que les estimulan a iniciar o mantener la intención de cambio a través de diferentes formas: llamado de atención, guía, acompañamiento, sugerencia o estímulo.

Por otro lado, Fleury y Moore (9) señalan que personas con enfermedad cardiovascular y baja actividad social tuvieron significativamente altos índices de mortalidad en comparación con aquellos que se mantenían activamente sociales.

Análisis de los ítems de la escala ASA en personas hospitalizadas con Hipertensión Arterial menos calificados

En general, los participantes del estudio pocas veces pueden tomar tiempo para su cuidado, esto se corroboró con la afirmación "con la actividad diaria puedo tomar tiempo para cuidarme" lo que puede ser explicado por la falta de tiempo debido al trabajo u ocupaciones y el vivir a las carreras, generado por la cambiante sociedad. Dentro de los componentes de poder Orem, que se ve comprometido en las personas con HTA es aquel que hace referencia a "La integración de las operaciones de autocuidado con otros aspectos de la vida".

Otro de los problemas que se observó en las personas hospitalizadas con hipertensión es la insatisfacción del requisito universal de autocuidado, que hace alusión al mantenimiento del equilibrio entre la actividad y el reposo, los datos arrojan que la mayoría de los participantes casi nunca o nunca pueden dormir lo suficiente para sentirse descansado. El descanso es considerado una acción que puede evitar el agotamiento, el estrés y que proporcionan salud mental, además que está muy relacionado con el componente de poder "uso controlado de la energía física", para poder iniciar y mantener una buena práctica de autocuidado.

Con respecto a la pregunta "pocas veces pienso en revisar mi cuerpo para saber si hay algún cambio", se encontró que las personas hipertensas casi nunca o nunca piensan en revisar su cuerpo para saber si hay algún cambio. El cuidado de enfermería debe ir dirigido a la potencialización del componente de poder "máxima atención y vigilancia", mediante la educación de las condiciones internas y externas a las que el individuo debe estar atento para el autocuidado. 
Otro de los problemas en las personas con HTA esta relacionada con la práctica de actividad física, donde gran porcentaje de los participantes afirman que casi nunca o nunca realizan actividad física. Esto significa que en estos individuos esta afectado los componentes de poder "Toma de decisiones sobre autocuidado y la operacionalización de estas decisiones" y "Adquisición, conservación y operacionalización técnica sobre autocuidado".

Lo anterior esta relacionado con el componente de poder señalado por Orem, que hace referencia al con el Uso controlado de la energía, el cual se encontró comprometido en las personas con hipertensión arterial, al afirmar "Con frecuencia no tengo la energía para cuidarme como debo".

Prieto R. encontró complementariedad en la relación existente entre el sedentarismo y la enfermedad crónica; el sedentarismo y el desconocimiento de prácticas saludables; los problemas de género y la actividad física. Este problema tiene importancia si se observa que las enfermedades crónicas presentan una alta asociación con el sedentarismo, el cual genera un riesgo atribuible a enfermedad cardiovascular de dos veces mayor que alguien activo. La autora identifico tres aspectos específicos de la problemática global: a) la falta de conocimiento y formación en prácticas de auto cuidado; b) el uso inadecuado del tiempo libre; y, c) la débil participación y vinculación del uso del tiempo libre y auto cuidado (10).

Se observa que la mayoría de los participantes tienen dificultades para alimentarse de manera que su peso se mantenga en lo correcto, las barreras que presentan los participantes para poder controlar su peso de forma adecuada, son las provocaciones, el gusto y los hábitos alimenticios. El ejercicio y la dieta, es la combinación perfecta para atacar las dos causas del sobrepeso y la obesidad: el exceso de ingesta calórica y el gasto calórico inadecuado.

Figueroa (11) concluye que a pesar de que las madres y su educación son el factor principal de los cuidados, se hace necesario el apoyo de toda la familia al ser ésta la unidad social económica de mayor responsabilidad para el bienestar nutricional; esto hace imprescindible considerar las funciones, conocimientos, limitaciones de tiempo, ingresos y recursos y motivaciones de los miembros del hogar y de la familia.• 


\section{REFERENCIAS}

1. Buselli EF. Stuart EM. Influence of psychosocial factors and biopsychosocial interventions on outcomes after myocardial infarction. Department of sociology, Ohio State University, Columbus, USA. Journal Cardiovascular Nursing. 1999; 13 (3): 60- 72.

2. Orem DE. Soins infirmiers: les concepts et la practique. Traducido por: Gosselin Danielle. Montreal: Décarie Éditeur inc; 1987.

3. Orem DE. Modelo de Orem. Conceptos de enfermería en la práctica. Barcelona: Masson Salvat; 1993.

4. Donohue J. Teoría del déficit de autocuidado. En: Marriner A, Raile M. Modelos y teorías de enfermería. Madrid: Harcourt Brace; 1994.

5. Isenberg A. Teoría de enfermería del déficit del autocuidado (TEDAC): Direcciones para avanzar la ciencia de enfermería y la práctica profesional. En: Parker M. Nursing Theories and Nursing practice. Philadelphia: F.A. Davis co; 2001. p.125.

6. Gast H, Denyes M, Campbell J, Hartweg D, Schott- Baer D, Isenberg M. Self-care agency: conceptualizations and operationalizations. Adv Nurs Sci 1989; 12 (1): 26-38.

7. Gallegos CE. Validez y confiabilidades de la versión en español de la escala: Valoración de las Capacidades de Autocuidado. Desarrollo Científ Enferm. 1998; 6 (9): 260-266.

8. Donohue J. Teoría del déficit de autocuidado. En: Marriner A, Raile M. Modelos y teorías de enfermería. Madrid: Harcourt Brace; 1994.

9. Moser DK. Maximing therapy in the advanced Herat failure patient. The Journal of cardiovascular Nursing. 1996; 10 (2): 29- 37.

10. Fleury J, Moore SM. Family - Centered Care after Acute Myocardial Infarction. The Journal of cardiovascular Nursing, 1999; 13 (3): 73- 82.

11. Prieto A. Modelo de Promoción de la Salud, con Énfasis en Actividad Física, para una Comunidad Estudiantil Universitaria. Rev. salud pública. 2003; 5 (3):284300.

12. Figueroa D. Estado Nutricional como Factor y Resultado de la Seguridad Alimentaria y Nutricional y sus Representaciones en Brasil. Rev. salud pública. 2004; 6 (2): 140-155. 